\title{
LA LUTTE CHRÉTIENNE CONTRE LE POUVOIR MUSULMAN EN OCCIDENT OU L'ORIGINE IBÉRIQUE DE LA CROISADE D'ORIENT
}

\author{
Por \\ GEORGES REYNAUD
}

Les croisades d'Orient ne sont qu'un épisode de la lutte que se livrèrent la Croix et le Croissant dans le monde médiéval. Pendant le Xle siècle, il n'y eut pas moins de quatorze expéditions françaises en Espagne et l'on s'accorde à trouver dans l'expédition qui prit BARBASTRO (1064-1065) la première véritable «croisade». L'expédition était en effet internationale, avec un élément primordial français et avait reçu une prédication apostolique (1). Faire remonter une attitude croisadiste à l'action de Charlemagne lors de la création de la marche d'Espagne ou au Comte Raymond Il de Rouergue «marquis de GOTHIE» qui combattit au-delà des Pyrénées en 985 serait un peu hasardeux, même si ce noble combattant fit à son retour don de son riche butin à l'abbaye de CONQUES (2). En 1087, Raymond de St. Guilles, Comte de Toulouse, avait, lui aussi, conduit des Provençaux et des Languedociens en expédition contre les musulmans d'Espagne, mais l'action déterminante dans le déclenchemet de la lutte chrétienne contre la puissance musulmane dans le nord de l'Espagne sera le fait des clunisiens!

Tout commença quan l'évèque de PAMPELUNE, SANCHE, impressionné par la renommée de Cluny décida de rejoindre la grande et prestigieuse abbaye. Le roi de Navarre et Aragon, prénommé également SANCHE et qualifié de «le Grand», informé de cette démarche d'adopter la réforme clunisienne et, dans ce but, envoya à CLUNY un moine de l'abbaye SAN JUAN DE LA PENA, PATERNE. Quelques années plus tard sur les instances de ce même roi et avec l'accord de l'abbé de CLUNY, ODILON, Paterne revint en Espagne et devint prieur de St. Jean de la Pen̂a.

(1) ANOUAR HATEM: «Les poèmes épiques des Croisades», París, GEUTHNER, 1932, p. 52.

(2) DOM. VAISSETTE: Hist. du Languedoc III, p. 198. 
Dès lors, le roi put compter sur l'aide totale de l'ordre de France y compris pour agrandir son territoire. En 1023, les évêques espagnols réunis à Pampelune décidèrent que les prélats de la ville seraient toujours choisis dans un monastère réformé par CLUNY. Le monastère de St Victorien éait reconstruit et peuplé de clunisiens. On convint aussi que ce serait parmi les moines de cet ordre que serait choisi l'évêque d'Aragon.

Le prestige des hommes de Cluny était sans égal (3) et il sanctionnait les résultats obtenus par des moines qui étaient aussi des hommes d'action, qui avaient déclaré la guerre légitime quand elle avait pour but d'exterminer les infidèles. Pendant deux cents ans l'ordre bourguignon ne cessa de régenter la papauté, gouvernant les rois, disciplinant l'ardeur belliqueuse des seigneurs, proclamant, au mépris de tous les puissants, la primauté du Spirituel, réalisant enfin le rêve de JEAN VIII, cette unité de l'Europe qu'un clunisien, URBAIN II, allait diriger vers l'Orient (4).

La captivité de MAYEUL, abbé de CLUNY, capturé par les sarrazins le 21 juillet 983 alors qu'il redescendait les pentes du Montgenèvre, avait grandement sensibilisé l'ordre et les puissants du sud de la France dans la lutte contre les musulmans. Le compte Guillaume I de Provence réunit une «croisade provençale» qui chassa les sarrazins de leurs repaires des Maures tels: La Garde-Freinet d'où ils attaquaient les pélerins se rendant à ROME.

CLUNY s'était, il est vrai, employé à la rénovation des quatre pélerinages, Rome, St Jacques de Compostelle, St Michel du Mt CARGAN (5) et le ST SÉPULCRE. Charlemagne, dans son voyage imaginaire vers le ST SÉPULCRE, se serait, dit-on, arrêté au Mt GARGAN pour implorer Saint Michel. La reconquête de la SICILE par les Normands fut une espèce de croisade, les normands ayant envahi l'Italie du Sud après leur pélerinage au Mt GARGAN en 1016.

C'est dans ce contexte de puissance clunisienne que les successeurs du roi SANCHE continuèrent à chercher l'appui des religieux français, notamment Ferdinand I de CASTILLE et son fils ALPHONSE VI. Sous son règne, les clunisiens se répandirent dans une grande partie de l'Espagne; l'un des chefs de la croisade de BARBASTRO aurait été un parent de l'abbé de Cluny. Les clunisiens auraient également poussé certains seigneurs français à aider ALPHONSE VI à conquérir Tolède, qui reçut pour archevêque un moine de la grande abbaye. L'épouse du roi, CONSTANCE, était une soeur d'un abbé de la communauté bourguignonne.

Comme support à la réception d'une aide venue de France, l'Espagne bénéficiait de la situation du grand pélerinage médiéval de St Jacques de Compostelle. On y venait de toute la chrétienté. Le sanctuarie avait été pris par les arabes d'AL MANSOUR le 10 août 997 mais le tombeau de St Jacques avait été respecté. Les clunisiens avaient le culte de St Jacques en grande estime. Les prieurs ODILON (6)

(3) Cf. M. PACAUT: «L'ordre de CLUNY", Paris, 1986; et aussi J. PRAWER: «Histoire du Royaune Latin de Jérusalem», +1, PARIS, CNRS, 1969, p. 167; "Le monastère de Cluny était alors le coeur de la spiritualité européenne ses abbés exerçaient un rôle politique considérable en Occident».

(4) A. HATEM, op. cit., p. 45.

(5) Ce pélerinage quelque peu oublié était si célèbre au Moyen Age qu'on donna le nom de Mt GARGAN à une crête du Limousin.

(6) ODILON DE MERCOEUR (994-1049). 
et HUGUES (7) s'étaient occupés du sanctuaire et avaient envoyé un des leurs, DALMACIUS, à Compostelle depuis quelques années. Quand le roi ALPHONSE VI lui confia la charge d'évêque, le pélerinage rentra dans l'orbite des clunisiens. Lorsque URBAIN II vint prêcher la croisade en France, DALMACIUS franchit les Pyrénées et se rendit auprés de lui. II obtint que les évêques de COMPOSTELLE, comme les abbés de CLUNY ne relèvent que du Saint Siège et que tous soient sacrés par le Pape. PASCAL II, successeur d'URBAIN et clunisien comme lui, fit montre à l'égard de Compostelle de la même sollicitude. En 1104, il accorda le «pallium» aux évêques de Compostelle à titre perpétuel et leur conféra de nombreux privilèges ecclésiastiques.

Les successeurs de Pascal II, GELASE II et CALIXTE \| étaient également clunisiens; ils eurent à l'égard de Compostelle la même sollicitude; Calixte II éleva le liu du pélerinage à la dignité de «Métropole».

CLUNY, écrit A. HATEM, «constitue l'un des liens qui forment l'unité de toutes les croisades françaises du Moyen-Age (8). Si les moines bourguignons exaltèrent le pélerinage de Compostelle, ils exaltèrent aussi le pélerinage du ST SÉPULCRE, ainsi qu'on peut le reconnaître dans les écrits de RAOUL GLABER qui souligne que le St Sépulcre attirait des milliers de gens de toutes origines e t de toutes conditions, pauvres et nobles unis dans une même ferveur (9).

Le rôle de CLUNY dans le débats du concile de CLERMONT (18 novembre 1095) fut très important. Urbain II séjourna une semaine dans la grande abbaye avant de gagner l'Auvergne. II eut de longs entretiens avec l'abbé HUGUES qui fut, peut-être, présent á Clermont avec d'autres clunisiens tels Jean de GAETE chef de la chancellerie romaine, DALMACIUS, évêque de Compostelle, Bernard de SIDERAC, primat de TOLEDE, Bérenger de TARRAGONE. Ceux-ci côtoyaient les plus importants prélats de France, Richard de ST VICTOR, Geoffroy de VENDOME, Landri de MACON, Lambert d'ARRAS. Au cours du concile qui n'avait pas été convoqué spécialement pour décider de la croisade (il concernait surtout la discipline intérieure de l'Eglise) Urbain II fit un éloge éclatant de CLUNY.

Cluny entretenait des relations étroites avec de grands féodaux qui comme Raymond de St Guilles combattirent en Espagne. Godefroy de Bouillon avait emmené des moines clunisiens avec lui et leur donnera un prieuré dans la vallée de JOSAPHAT. Cluny reste toujours le moteur des pélerinages et les croisés ou leurs chefs suivent de près les pélerins. «Rien ne distingue, essentiellement, les croisades d'Orient de celles d'Espagne, d'Italie ou de Sicile»; un pélerinage a toujours précédé une croisade au moment du concile de CLERMONT c'est peut-être la croisade en ...ESPAGNE! qui préoccupait le plus URBAIN II ainsi que l'écrit GUIBERT de NOGENT (10). L'inquiétude du pape venait de l'invasion des ALMORAVIDES en Espagne, le sort des chrétiens de la Péninsule était certainement plus pressant que le sort des chrétiens de Syrie. De nombreux croisés français avaient trouvé la mort lors de la défaite

(7) HUGUES DE SEMUR (1049-1109).

(8) A. HATEM, op. cit., p. 63.

(9) RAOUL GLABER Vl, 6, ed. PROU, p. 106.

(10) Rec des Hist. des Croisades, Hist. Occ. IV, p. 135 
de ZALACCA (1087) contre les ALMORAVIDES (11). Le péril semblait menaçant aux marches de la Chrétienté. Une «croisade» s'organisa en France à la suite de cet événement dès 1087. Plus tard (1er juillet 1089) et après s'être assuré de la fidélité des normands d'Italie, le pape assura les positions chrétiennes en Espagne. En 1089 il garantissait à BERANGER, Comte de Barcelone, à ERMENGAUD Comte d'URGEL, au Comte de BESALU Bernard et à tous les évêques et nobles des provinces de Tarragone et Barcelone la même indulgence que celle accordée aun pélerinage de Jérusalem s'ils contribuaient à renforcer les défenses de Tarragone qui devait être un rempart contre les indifèles. Le chroniqueur anglais Guillaume de MALMESBURY nous montre le pape occupé par les affaires d'Espagne (13) et ORDERIC VITAL affirme qu'il pensait à l'Afrique rappelant dans un discours que les Sarrazins y ont détruit 96 épiscopats. Cette attitude est corroborée par IBN AL ATHIR qui, au début de sa relation, affirme que les croisés s'étaient réunis pour envahir l'Afrique (14). Ce serait ROGER DE SICILE qui, craignant pour ses états, aurait détourné l'expédition vers les lieux saints. L'idée d'une conquête de l'Afrique sera reprise par Saint Louis et les croisés de Barbarie (1365) ramèneront l'intérêt sur cette aire géographique.

L'Influence de la lutte contre l'Islam en Espagne est à notre sens indéniable dans la formation de la mentalité «croisadiste» qui a dirigé des centaines de milliers «d'Occidentaux» vers l'Orient. Bien que ce point ait été quelque peu négligé par les grands historiens des croisades, il est, á notre avis, essentiel.

Cette influence ibérique n'avait pas échappé à l'attention d'historiens tels Etienne DELARUELLE ou JOSHUA PRAWER que nous citons ici in extenso: «si l'on ne peut assurer que la visite du pape à CLUNY fut un élément déterminant dans la maturation de l'idée de croisade Cluny joua effectivement un rôle important dans les "croisades" d'Espagne, et, comme ses maisons étaient nombreuses en ce pays, le pape aurait pu recevoir dans la capitale clunisienne un rapport sur la situation en Espagne. Mais on manque d'information sur les liens de Cluny avec l'Orient. Cependant il n'est pas impossible que l'esprit des «croisades» espagnoles, vivace dans ce couvent, ait contribué à éclairer les idées du pape. A supposer que, dès son arrivée à France, le pape eût nourri le projet d'une entreprise chrétienne dans l'Orient byzantin, ce contact avec les Clunisiens, artisans de l'entreprise espagnole de «Reconquista", et avec des personnalités qui pouvaient le renseigner sur la situation dans l'Islam espagnol et oriental, Asie Mineure et Terre Sainte, permit que s'élaborât l'idée de Croisade que se précisât son organisation, et que son principal objectif fût déterminé» (15).

Dans le même sens nous citerons $M$. Marius CANARD: «On sait d'autre part que le rôle de l'ordre dans les croisades d'Espagne et de Sicile fut prépondérant; de même, on connaît son influence sur les papes GREGOIRE VII et URBAIN II, dont le pon-

(11) DOZY: «Histoire des Mulsumans d'Espagne», IV, p. 205-206.

(12) Au Concile d'AMALFI en 1088

(13) CREGUT: «Concile de Clermont appendice», p. 362.

(14) IBN AL ATHIR rapporté par F. GABRIELLI: «Chroniques arabes des Croisades», PARIS, SIND BAD, 1977, p. 25.

(15) J. PRAWER, op. cit., ibid, T. I, p. 167. 
tificat fut décisif pour les croisades d'Orient» (16).

Basée sur l'exaltation du pélerinage et la lutte pour la foi chrétienne, l'action de l'abbaye bourguignonne de Cluny, magnifiée par URBAIN II débouchera sur l'exaltation de la libération du ST SÉPULCRE et favorisera le départ en masse (4 armées) des occidentaux pour la première croisade. Ainsi, à la suite d'une longue maturation idéologique sur laquelle la situation de l'Occident Musulman a une part certaine les hommes de l'Ouest vont se dirier vers Jérusalem, objet lointain chargé de tans de voeux et de tans d'espoirs.

(16) M. CANARD: «La guerre sainte dans le monde islamique et dans le monde chrétien», in Revue Africaine, $n .^{\circ}$ 79, ALGER, 1936, p. 614. 